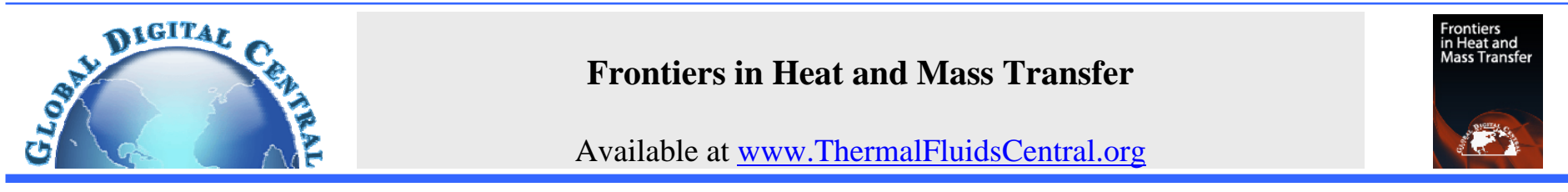

\title{
INFLUENCE OF PORE WALL SURFACE PROPERTY ON FLUX OF CYLINDRICAL-SHAPED NANOPOROUS FILTERING MEMBRANE
}

\author{
Yongbin Zhang* \\ College of Mechanical Engineering, Changzhou University, Changzhou, 213164, Jiangsu Province, China
}

\begin{abstract}
The influence of pore wall surface property on the flux of a novel cylindrical-shaped nanoporous filtering membrane is analytically studied by using the flow factor approach model for a nanoscale flow. Across the thickness of the membrane are manufactured two concentric cylindrical pores with different radii. The smaller nanoscale pore is for filtration, while the other larger pore is for reducing the flow resistance. It was found that when the larger pore wall surface is hydrophobic, the interaction between the filtered liquid and the smaller pore wall surface has a very significant effect on the value of the optimum ratio of the radii of these two pores which yields the smallest flow resistance and thus the highest flux of the membrane; In the optimum working condition, the smaller pore wall surface property greatly influences the flow resistance of the membrane. It was found to be possible to manufacture the membrane with specific pore wall surface property used for filtering one liquid out of other liquids; In this membrane, the pore wall surface should be hydrophobic to the filtered liquid, while it should be highly hydrophilic to the other liquids.
\end{abstract}

Keywords: Membrane; Filtration; Nanopore; Flux; Interaction; Model

\section{INTRODUCTION}

Nanoporous membranes have been invented for ultimate filtration because of their nanoscale filtering pores (Hillmyer et al., 2015; Tringe et al., 2013). They can be used for super purification of water, hemofiltration, DNA analysis and drug delivery (Das et al., 2014; Fissel et al., 2009; Han et al., 2013; Jackson and Hillmyer, 2010; Kim et al., 2006; Venkatesan et al., 2009). One of the problems of these membranes is their high flow resistances because of nanoscale confining pores, which can result in fairly small fluxes of the membranes. It was shown that this slow flow is due to the nanoscale pore radius, the increased effective viscosity of the liquid in the confining pores and the significant non-continuum effect of the liquid across the pore radius (Zhang, 2016). It was suggested that increasing the liquid temperature can effectively improve the flow rate through nanoscale pores (Zhang, 2017a).

People persist their efforts in finding the way to improve the fluxes of these membranes; The main direction was to reduce the flow resistance of the membrane by reducing the membrane thickness or/and partly enlarging the pores within the membrane. Surwade et al. (2015) found that a nanoporous membrane made of a mono layer graphene can yield a high membrane flux in water desalination. Li et al. (2004) found that a conical-shaped nanopore is advantageous over a cylindricalshaped nanopore for giving a higher flux through the membrane. Yang et al. (2006) proposed a mixed membrane in which the top layer has cylindrical nanopores while the bottom layer is a conventional microfiltration membrane.

In the former study (Zhang, 2017b), a novel cylindrical-shaped nanoporous filtering membrane was proposed. Across the thickness of this membrane are manufactured two concentric cylindrical pores with different radii; The smaller nanoscale pore is for filtration, while the other larger pore is for reducing the flow resistance. The optimum ratio between the radii of these two pores was found for yielding the smallest flow resistance of this membrane (Zhang, 2017b). It was found that this optimum ratio is strongly influenced by the radius and depth of the filtration pore and the interaction between the filtered liquid and the pore wall surface. In that study, the surface properties of the two pore walls were considered as identical.

As a continuous research, the present paper analytically studies the performance of the cylindrical-shaped nanoporous filtering membrane proposed by Zhang (2017b) by taking the surface properties of the two pore walls as different. In the present study, the surface of the larger pore wall is highly hydrophobic to the filtered liquid, while the interaction between the filtered liquid and the smaller pore wall can be weak, medium-level or strong. The influences of the surface property of the filtration pore wall on the optimum ratio of the radii of these two pores and the flow resistance of the membrane are thus studied. On the other hand, the function of the membrane for filtering one liquid out of other liquids is studied considering different liquid-pore wall interactions among the mixed liquids.

\section{STUDIED MEMBRANE}

The studied membrane is shown in Fig.1. The configuration of this membrane is same as shown in the study by Zhang (2017b). The filtering nanopores with the radius $R_{0}$ are uniformly manufactured on the surface of the membrane, and across the membrane thickness the larger concentric pores with the radius $R_{1}$ are manufactured for reducing the flow resistance. The axial lengths (i.e. depths) of the two pores are respectively $l_{0}$ and $l_{1}$, and the membrane thickness is $l$. The difference of this membrane from the membrane studied by Zhang (2017b) is that the surface properties of the two pore walls across the 
membrane thickness may be not identical, the surface of the larger pore wall is highly hydrophobic to the filtered liquid, while the surface of the smaller pore wall may be hydrophobic or hydrophilic to the filtered liquids; Or, although the pore wall surface properties may be identical, the surfaces of the two pore walls have greatly different interactions with the liquids which are mixed together; In this case, the membrane may be able to be used for filtering one liquid out of the other liquids because of largely different flow resistances of the membrane for different liquids.

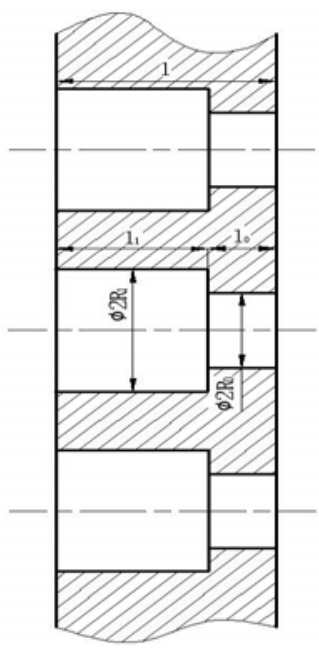

Fig. 1 The studied nanoporous filtering membrane (Zhang, 2017b).

\section{ANALYSIS}

\subsection{Basic Equations}

The analysis for the membrane in Fig.1 has been developed in the study by Zhang (2017b). Here are only repeated the necessary analytical results. The mass flow rate (i.e. the flux) through the membrane is (Zhang, 2017b):

$q_{m}=\frac{\Delta p}{i_{f}}$

where $\Delta p$ is the pressure drop across the membrane thickness and $i_{f}$ is termed as the flow resistance.

The flow resistance $i_{f}$ of the membrane can measure the flux capability of the membrane. For a given $\Delta p$, a lower $i_{f}$ gives a higher flux of the membrane, vice versa. $i_{f}$ is expressed as (Zhang, 2017b):

$i_{f}=\frac{4 \eta}{\pi \rho R_{0}^{3}} I_{f}$

where $\rho$ and $\eta$ are respectively the bulk density and the bulk viscosity of the filtered liquid at the environmental temperature and pressure, and the dimensionless flow resistance $I_{f}$ is:

$I_{f}=F\left(\frac{R_{1}}{R_{0}}\right) \frac{\pi R_{0}^{2} \lambda_{1}}{\chi A_{m}}$

Here, $\lambda_{l}=l / R_{0}, \quad \chi$ is the pore production rate of the membrane surface, $A_{m}$ is the area of the surface of the membrane, and the function $F\left(R_{1} / R_{0}\right)$ is (Zhang, 2017b):

$F\left(\frac{R_{1}}{R_{0}}\right)=\frac{\lambda_{0} \operatorname{Cy}\left(\bar{R}_{0}\right)}{\operatorname{Cq}\left(\bar{R}_{0}\right)\left|S\left(\bar{R}_{0}\right)\right|}\left(\frac{R_{1}}{R_{0}}\right)^{2}+\frac{\left(1-\lambda_{0}\right) \operatorname{Cy}\left(\bar{R}_{1}\right)}{\operatorname{Cq}\left(\bar{R}_{1}\right)\left|S\left(\bar{R}_{1}\right)\right|\left(\frac{R_{1}}{R_{0}}\right)^{2}}$

where $\lambda_{0}=l_{0} / l, \quad \bar{R}_{0}=R_{0} / R_{c r, 0}, \quad \bar{R}_{1}=R_{1} / R_{c r, 1}, \quad R_{c r, 0} \quad$ and $R_{c r, 1}$ are respectively the critical radii of the smaller and larger pores for the liquid to become continuum across the pore radius, $C y(\bar{R})=\eta_{b f}^{\text {eff }}(\bar{R}) / \eta, \quad C q(\bar{R})=\rho_{b f}^{\text {eff }}(\bar{R}) / \rho, \quad \rho_{b f}^{\text {eff }} \quad$ and $\eta_{b f}^{\text {eff }}$ are respectively the average density and the effective viscosity of the confined liquid across the pore radius, and $S$ is the parameter depicting the non-continuum effect of the confined liquid across the pore radius $(-1 \leq S<0)$.

\subsection{Parametric Optimization}

For attaining a highest flux of the membrane, the flow resistance of the membrane should be the smallest. For achieving this goal, the radius $R_{1}$ should be so designed that it yields the smallest value of the function $F\left(R_{1} / R_{0}\right)$.

When the wall surface of the larger pore with the radius $R_{1}$ is highly hydrophobic to the filtered liquid, $C y\left(\bar{R}_{1}\right) \approx 1, C q\left(\bar{R}_{1}\right) \approx 1$ and $\left|S\left(\bar{R}_{1}\right)\right| \approx 1$. In this condition, the optimum ratio of $R_{1}$ to $R_{0}$ for yielding the smallest $F\left(R_{1} / R_{0}\right)$ is (Zhang, 2017b):

$$
\left(\frac{R_{1}}{R_{0}}\right)_{\text {opt }}= \begin{cases}{\left[\frac{\left(1-\lambda_{0}\right) C q\left(\bar{R}_{0}\right)\left|S\left(\bar{R}_{0}\right)\right|}{\lambda_{0} \operatorname{Cy}\left(\bar{R}_{0}\right)}\right]^{\frac{1}{4}}} & , \text { for } R_{1} \geq R_{0} \\ 1 & , \text { for } R_{1}<R_{0}\end{cases}
$$

The resulting minimum value of $F\left(R_{1} / R_{0}\right)$ is (Zhang, 2017b):

$$
F_{\min }= \begin{cases}2 \sqrt{\frac{\lambda_{0}\left(1-\lambda_{0}\right) C y\left(\bar{R}_{0}\right)}{C q\left(\bar{R}_{0}\right)\left|S\left(\bar{R}_{0}\right)\right|}} & \text { for } R_{1} \geq R_{0} \\ \frac{C y\left(\bar{R}_{0}\right)}{C q\left(\bar{R}_{0}\right)\left|S\left(\bar{R}_{0}\right)\right|} & \text { for } R_{1}<\mathrm{R}_{0}\end{cases}
$$

In the present study, the optimum $R_{1} / R_{0}$ value and the minimum value of $F\left(R_{1} / R_{0}\right)$ can respectively be accurately calculated from Eqs. (5) and (6) whenever the interaction between the filtered liquid and the wall surface of the filtering pore (with the radius $R_{0}$ ) is weak, medium-level or strong. However, as pointed out by Zhang (2017b), if the wall surface properties of the two pores are identical, equations (5) and (6) both may be not accurate for respectively calculating the optimum $R_{1} / R_{0}$ value and the minimum value of $F\left(R_{1} / R_{0}\right)$ when the liquid-pore wall interaction is mediumlevel or strong.

Equation (6) shows that in the present study, the minimum value of $F\left(R_{1} / R_{0}\right)$ i.e. the smallest flow resistance of the membrane is intimately related to the interaction between the filtered liquid and the 
filtering pore wall (reflected by the parameters $\operatorname{Cy}\left(\bar{R}_{0}\right), C q\left(\bar{R}_{0}\right)$ and $\left.S\left(\bar{R}_{0}\right)\right)$.

\section{EXEMPLARY CALCULATIONS}

Exemplary calculations were carried out for investigating the influence of the hydrophobic or hydrophilic surface properties of the filtering pore wall on the optimum $R_{1} / R_{0}$ value and the corresponding smallest flow resistance of the membrane. In the calculation, the interaction between the filtered liquid and the filtering pore wall can be weak, medium-level or strong.

For whichever liquid-pore wall interaction, $C q(\bar{R})$ is generally expressed as (Zhang, 2014; Zhang, 2017b):

$C q(\bar{R})=\left\{\begin{array}{l}1 \quad \text { for } \quad \bar{R} \geq 1 \\ m_{0}+m_{1} \bar{R}+m_{2} \bar{R}^{2}+m_{3} \bar{R}^{3} \quad \text {, for } 0<\bar{R}<1\end{array}\right.$

where $\bar{R}$ can be $\bar{R}_{0}$ or $\bar{R}_{1}$ (same in the following equations), $m_{0}$, $m_{1}, m_{2}$ and $m_{3}$ are respectively constants.

$C y(\bar{R})$ is generally expressed as (Zhang, 2014; Zhang, 2017b):

$C y(\bar{R})=\left\{\begin{array}{lll}1 \quad & \text { for } \quad \bar{R} \geq 1 \\ a_{0}+\frac{a_{1}}{\bar{R}}+\frac{a_{2}}{\bar{R}^{2}} \quad, & \text { for } 0<\bar{R}<1\end{array}\right.$

where $a_{0}, a_{1}$ and $a_{2}$ are respectively constants.

$S(\bar{R})$ is generally expressed as (Zhang, 2014; Zhang, 2017b):

$S(\bar{R})=\left\{\begin{array}{lll}-1 & \text { for } \quad \bar{R} \geq 1 \\ {\left[n_{0}+n_{1}\left(\bar{R}-n_{3}\right)^{n_{2}}\right]^{-1}} & , \text { for } n_{3}<\bar{R}<1\end{array}\right.$

where $n_{0}, n_{1}, n_{2}$ and $n_{3}$ are respectively constants.

For weak, medium-level and strong filtered liquid-filtering pore wall interactions, the values of $R_{c r, 0}$ were respectively taken as $3.5 \mathrm{~nm}$, 10nm and 20nm (Zhang, 2014; Zhang, 2017b). For different types of the filtered liquid-filtering pore wall interaction, the values of the other parameters are respectively shown in Tables $1(\mathrm{a}-\mathrm{c})$.

Table 1(a) Liquid viscosity data for different liquid-pore wall interaction types (Zhang, 2014; Zhang, 2017b)

\begin{tabular}{|l|c|c|c|}
\hline Interaction & $a_{0}$ & $a_{1}$ & $a_{2}$ \\
\hline Strong & 1.8335 & -1.4252 & 0.5917 \\
\hline Medium & 1.0822 & -0.1758 & 0.0936 \\
\hline Weak & 0.9507 & 0.0492 & $1.6447 \mathrm{E}-4$ \\
\hline
\end{tabular}

Table 1(b) Liquid density data for different liquid-pore wall interaction types (Zhang, 2014; Zhang, 2017b)

\begin{tabular}{|l|r|r|r|c|}
\hline Interaction Parameter & $m_{0}$ & $m_{1}$ & $m_{2}$ & $m_{3}$ \\
\hline Strong & 1.43 & -1.723 & 2.641 & -1.347 \\
\hline
\end{tabular}

\begin{tabular}{|l|c|c|c|c|}
\hline Medium & 1.30 & -1.065 & 1.336 & -0.571 \\
\hline Weak & 1.116 & -0.328 & 0.253 & -0.041 \\
\hline
\end{tabular}

Table 1(c) Liquid non-continuum property data for different liquid-pore wall interaction types (Zhang, 2014; Zhang, 2017b)

\begin{tabular}{|l|c|c|c|c|}
\hline Interaction Parameter & $n_{0}$ & $n_{1}$ & $n_{2}$ & $n_{3}$ \\
\hline Strong & 0.4 & -1.374 & -0.534 & 0.035 \\
\hline Medium & -0.649 & -0.343 & -0.665 & 0.035 \\
\hline Weak & -0.1 & -0.892 & -0.084 & 0.1 \\
\hline
\end{tabular}

The calculations were also carried out for investigating the possibility of the membrane used for filtering one liquid out of other liquids (i.e. for a liquid-liquid separation). In these calculations, the surface properties of the two pore walls were taken as identical, the flow resistances i.e. the values of the function $F\left(R_{1} / R_{0}\right)$ of the membrane for three liquids were respectively calculated (by using Eq. (4)) when these liquids i.e. Liquid A, Liquid B and Liquid C respectively have weak, medium-level and strong interactions with the pore wall surfaces. In these calculations, $R_{c r, 0}=R_{c r, 1}$, and the values of the parameters for formulating $C y(\bar{R}), C q(\bar{R})$ and $S(\bar{R})$ (Here, $\bar{R}$ is $\bar{R}_{0}$ or $\bar{R}_{1}$ ) are same as shown above for each kind of liquid-pore wall interaction.

\section{RESULTS AND DISCUSSION}

\section{1 For Different Surface Properties Of The Two Pore Walls}

As stated above, in this calculation, the wall surface of the pore with the radius $R_{1}$ is highly hydrophobic to the filtered liquid, and the wall surface of the filtering pore with the radius $R_{0}$ may be hydrophobic or hydrophilic.

Figures 2(a) and (b) respectively show the influences of the interaction between the filtered liquid and the filtering pore wall on the optimum $R_{1} / R_{0}$ value when $\lambda_{0}=0.001$ and $\lambda_{0}=0.01$. When $\bar{R}_{0} \leq 0.7$, the optimum $R_{1} / R_{0}$ value is shown to be significantly reduced with the increase of the interaction strength between the filtered liquid and the filtering pore wall. However, when $\bar{R}_{0} \geq 0.8$, the surface (hydrophobic or hydrophilic) property of the filtering pore wall has a negligible influence on the optimum $R_{1} / R_{0}$ value. When $\bar{R}_{0} \geq 0.9$, the optimum $R_{1} / R_{0}$ values approach to the same constant in spite of the liquid-filtering pore wall interaction.

Figures 3(a) and (b) respectively show the correspondingly obtained minimum values of $F\left(R_{1} / R_{0}\right)$ i.e. $F_{\min }$ for different $\bar{R}_{0}$ and different filtered liquid-filtering pore wall interactions when $\lambda_{0}=0.001$ and $\lambda_{0}=0.01$. When $\bar{R}_{0} \leq 0.6$, the interaction between the filtered liquid and the filtering pore wall has a significant effect on the value of $F_{\text {min }}$ i.e. the flow resistance of the membrane. When $\bar{R}_{0} \leq 0.2$, the surface (hydrophobic or hydrophilic) property of the filtering pore wall has a great influence on the flow resistance of the membrane; For a low $\bar{R}_{0}$, the flow resistance of the membrane for a strong liquid-filtering pore wall interaction can be ten times of that for a weak liquid-filtering pore wall interaction. However, when $\bar{R}_{0} \geq 0.7$, the surface property of the filtering pore wall is shown to have a negligible influence on the flow resistance of the membrane. 


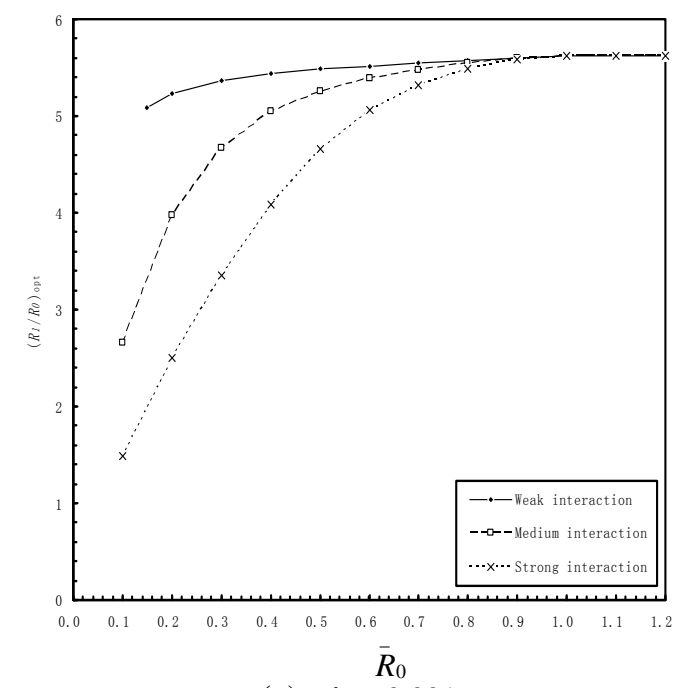

(a) $\lambda_{0}=0.001$

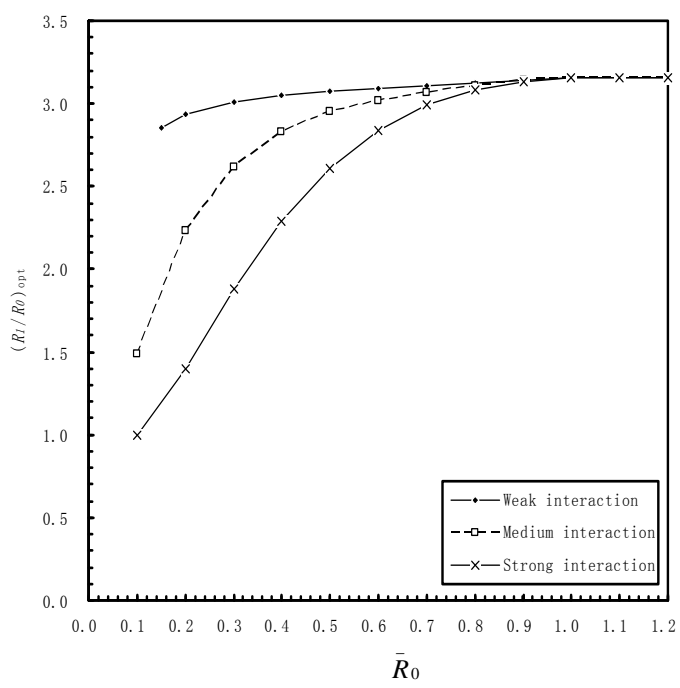

(b) $\lambda_{0}=0.01$

Fig. 2 Values of $\left(R_{1} / R_{0}\right)_{o p t}$ for different $\bar{R}_{0}$ and different filtered liquid-filtering pore wall interactions when $\lambda_{0}=0.001$ and $\lambda_{0}=0.01$

\subsection{For Different Liquids}

As stated above, in this calculation, the surface properties of the two pore walls are identical, while the three liquids i.e. Liquid A, Liquid B and Liquid $\mathrm{C}$ were respectively taken and they respectively have weak, medium-level and strong interactions with the pore wall surfaces. In this calculation, for a given $R_{0}$, the value of $R_{1}$ was determined as an optimum one by Eq. (5) according to the weak liquid-pore wall interaction i.e. according to Liquid A; However, for Liquids B and C, this determined $R_{1}$ value should not be the optimum for giving the smallest flow resistance of the membrane.

Figures 4(a) and (b) respectively plot the values of $F\left(R_{1} / R_{0}\right)$ calculated from Eq. (4) i.e. the dimensionless flow resistances of the membrane against $\bar{R}_{0}$ for Liquids $\mathrm{A}, \mathrm{B}$ and $\mathrm{C}$ when $\lambda_{0}=0.001$ and $\lambda_{0}=0.01$. In these figures, $\bar{R}_{0}=R_{0} / R_{c r, 0}$, and $R_{c r, 0}=3.5 \mathrm{~nm}$ determined for Liquid A. In the investigated $\bar{R}_{0}$ range, for the same operating condition, the differences among the flow resistances of the membrane respectively for Liquids A, B and C are very obvious. For $\bar{R}_{0} \leq 0.25$, for the same operating condition, the flow resistance of the membrane for Liquid $\mathrm{C}$ is more than one thousand times that for Liquid A; Even for $\bar{R}_{0}=0.5$, the flow resistance of the membrane for Liquid C is nearly one hundred times that for Liquid A. Such a difference for these two liquids is increased with the reduction of $\bar{R}_{0}$. These results provide the indication that the studied membrane can be used for filtering one liquid out of other liquids, provided that the pore wall surfaces are hydrophobic to the filtered liquid (such as Liquid A), while they are highly hydrophilic to the other liquids (such as Liquid C). For the best effect of a liquid-liquid separation, the radius $R_{0}$ of the filtering pore should be as small as possible.

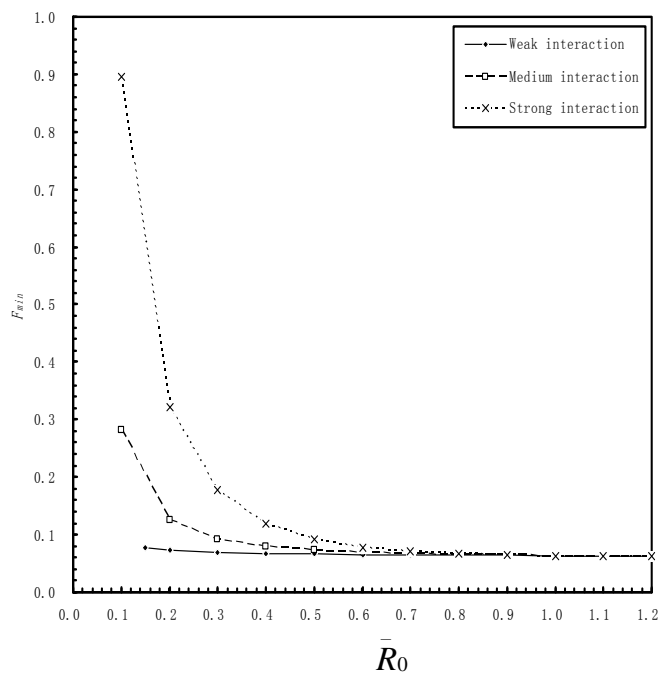

(a) $\lambda_{0}=0.001$

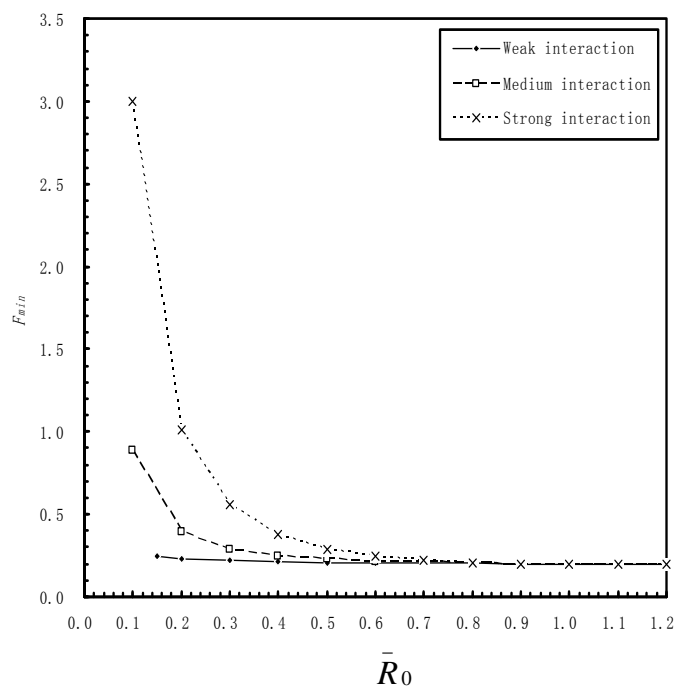

(b) $\lambda_{0}=0.01$

Fig. 3 Minimum values of $F\left(R_{1} / R_{0}\right)$ i.e. $F_{\text {min }}$ for different $\bar{R}_{0}$ and different filtered liquid-filtering pore wall interactions when the $R_{1} / R_{0}$ values are taken as optimum as respectively shown in Figs. 2(a) and (b).

\section{CONCLUSIONS}

The paper analytically investigates the influence of the pore wall surface property on the flow resistance i.e. the flux capability of the membrane in the novel cylindrical-shaped nanoporous filtering membrane proposed by Zhang (2017b), by using the flow factor approach model for a nanoscale flow. Across the thickness of this 
membrane are manufactured two concentric pores with different radii; The smaller nanoscale pore with the radius $R_{0}$ is for filtration, while the other larger pore with the radius $R_{1}$ is for reducing the flow resistance. The pores are uniformly distributed on the surface of the membrane.

In this study, first, the surface of the larger pore wall was taken as highly hydrophobic to the filtered liquid, and the influence of the surface property of the smaller (filtering) pore wall was investigated. It was found that the (hydrophobic or hydrophilic) surface property of the filtering pore wall has a significant influence on the optimum $R_{1} / R_{0}$ value which yields the smallest flow resistance of the membrane, when $\bar{R}_{0} \leq 0.7$. While the corresponding resulting smallest flow resistance of the membrane is significantly influenced by the surface property of the filtering pore wall when $\bar{R}_{0} \leq 0.6$. Here, $\bar{R}_{0}=R_{0} / R_{c r, 0}$, and $R_{c r, 0}$ is the critical radius of the filtering pore for the filtered liquid to become continuum across the pore radius.

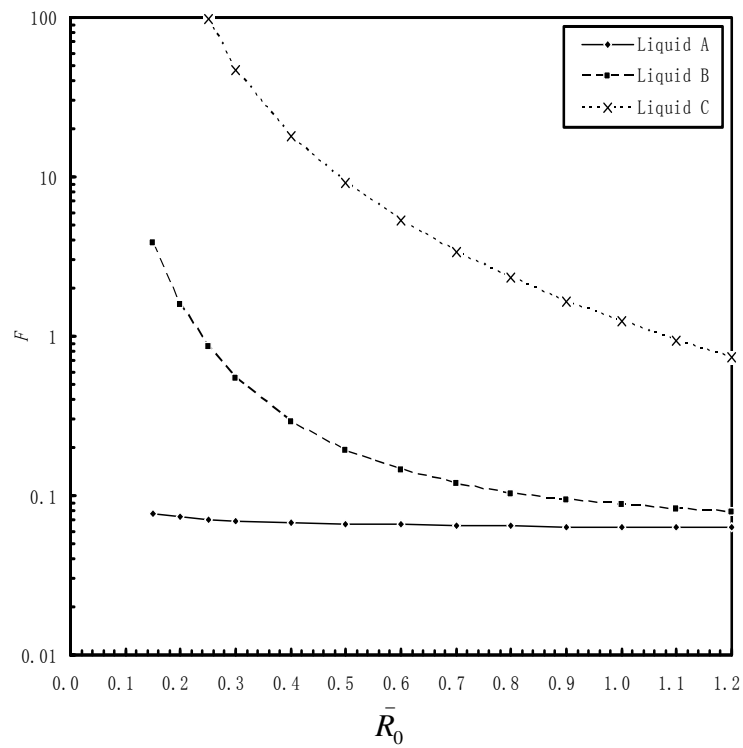

(a) $\lambda_{0}=0.001$

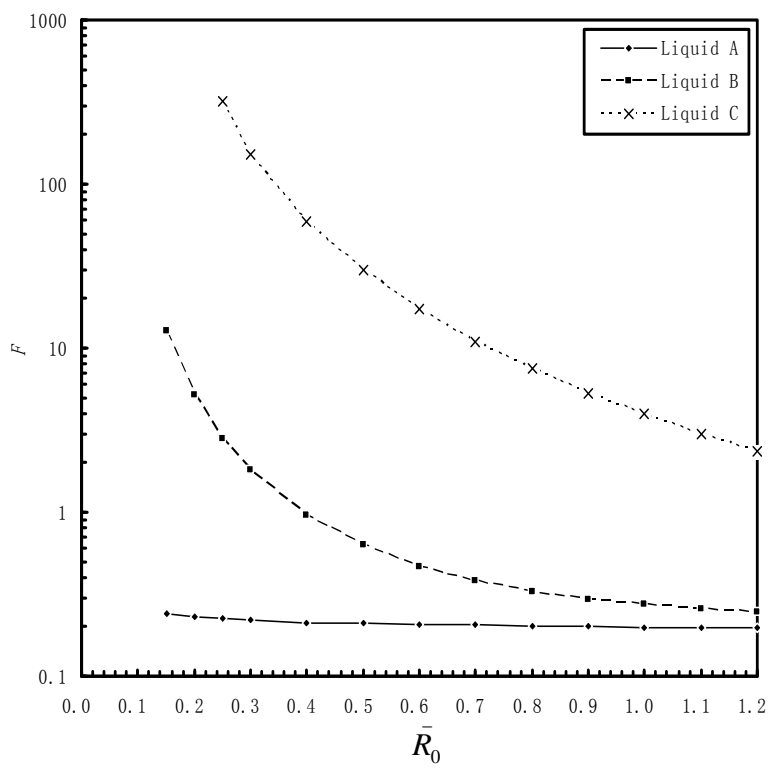

(b) $\lambda_{0}=0.01$

Fig. 4 Values of $F\left(R_{1} / R_{0}\right)$ calculated from Eq.(4) i.e. the dimensionless flow resistances of the membrane respectively for Liquids A, B and C.
Secondly, by taking the surface properties of the two pore walls as identical, the flow resistances of the membrane respectively for three liquids were investigated; these liquids respectively have weak, medium-level and strong interactions with the pore wall surfaces. It was found that when $\bar{R}_{0} \leq 0.5$, for the same operating condition, the flow resistance of the membrane for the exhibiting highly hydrophilic pore wall surface because of the liquid can be more than one hundred times or even more than one thousand times that for the exhibiting hydrophobic pore wall surface for another liquid. This provides an indication that the studied membrane can be used for filtering one liquid out of other liquids i.e. a liquid-liquid separation.

\section{REFERENCES}

Das, R., Eaqub Ali, Md, Abd Hamid, S. B., Ramakrishna, S., and Chowdhury, Z. Z., 2014, "Carbon Nanotube Membranes for Water Purification: A Bright Future in Water Desalination,” Desalination, 336, 97-109.

https://doi.org/10.1016/j.desal.2013.12.026

Fissel, W. H., Dubnisheva, A., Eldridge, A. N., Fleischman, A. J., Zydney, A. L., and Roy, S., 2009, "High-Performance Silicon Nanopore Hemofiltration Membranes,” Journal of Membrane Science, 326, 58-63. https://doi.org/10.1016/j.memsci.2008.09.039

Han, Y., Xu, Z., and Gao, C., 2013, "Ultrathin Graphene Nanofiltration Membrane for Water Purification,” Advanced Functional Materials, 23, 3693-3700. https://doi.org/10.1002/adfm.201202601

Hillmyer, M.A., Jackson, E.A., and Lee, Y., 2015, “Nanoporous Filtration Membranes,” US Patent App. 20150336058.

Jackson, E. A., and Hillmyer, M. A., 2010, "Nanoporous Membranes Derived from Block Copolymers: From Drug Delivery to Water Filtration,” ACS Nano, 4, 3548-3553.

https://doi.org/10.1021/nn1014006

Kim, M. J., Wanunu, M., Bell, D. C., and Meller, A., 2006, "Rapid Fabrication of Uniformly Sized Nanopores and Nanopore Arrays for Parallel DNA Analysis,” Advanced Materials, 18, 3149-3153. https://doi.org/10.1002/adma.200601191

Li, N., Yu, S., Harrell, C., and Martin, C. R., 2004, "Conical Nanopore Membranes: Preparation and Transport Properties," Analytical Chemistry, 76, 2025-2030. https://doi.org/10.1021/ac035402e

Surwade, S. P., Smirnov, S. N., Vlassiouk, I. V., Unocic, R. R., Veith, G. M., Dai, S., and Mahurin, S. M., 2015, "Water Desalination Using Nanoporous Single-Layer Grapheme,” Nature Nanotechnology, 10, 459-464.

https://doi.org/10.1038/nnano.2015.37

Tringe, J. W., Balhom, R. L., and Zaidi, S., 2013, “Nanoporous Membrane,” US Patent App. 20130306549.

Venkatesan, B. M., Dorvel, B., Yemenicioglu, S., Watkins, N., Petrov, I., and Bshir, R., 2009, "Highly Sensitive, Mechanically Stable Nanopore Sensors for DNA Analysis,” Advanced Materials, 21, 27712776.

https://doi.org/10.1002/adma.200803786

Yang, S. Y., Ryu, I., Kim, H. Y., Kim, J. K., Jang, S. K., and Russell, T. P., 2006, "Nanoporous Membranes with Ultrahigh Selectivity and Flux for the Filtration of Viruses," Advanced Materials, 18, 709-712. https://doi.org/10.1002/adma.200501500

Zhang, Y. B., 2014, “Lubrication Analysis for a Line Contact Covering from Boundary Lubrication to Hydrodynamic Lubrication: Part IMicro Contact Results,” Journal of Computational and Theoretical Nanoscience, 11, 62-70.

https://doi.org/10.1166/jctn.2014.3318 
Zhang, Y. B., 2016, “The Flow Equation for a Nanoscale Fluid Flow,” International Journal of Heat and Mass Transfer, 92, 1004-1008. https://doi.org/10.1016/j.ijheatmasstransfer.2015.09.008

Zhang, Y. B., 2017a, “Effect of Temperature on Water Transportation in Nanochannel," Frontiers in Heat and Mass Transfer, 9, 013016. https://doi.org/10.5098/hmt.9.16

Zhang, Y. B., 2017b, “Optimum Design for Cylindrical-Shaped Nanoporous Filtering Membrane," International Journal of Heat and Mass Transfer, submitted. 